\title{
The NMDA-to-AMPA Ratio at Synapses Onto Layer 2/3 Pyramidal Neurons Is Conserved Across Prefrontal and Visual Cortices
}

\author{
Chaelon I. O. Myme, Ken Sugino, Gina G. Turrigiano, and Sacha B. Nelson \\ Department of Biology, Volen Center for Complex Systems, Brandeis University, Waltham, Massachusetts 02454-9110
}

Submitted 27 January 2003; accepted in final form 28 March 2003

\begin{abstract}
Myme, Chaelon I. O., Ken Sugino, Gina G. Turrigiano, and Sacha B. Nelson. The NMDA-to-AMPA ratio at synapses onto layer $2 / 3$ pyramidal neurons is conserved across prefrontal and visual cortices. J Neurophysiol 90: 771-779, 2003. First published April 2, 2003; 10.1152/jn.00070.2003. To better understand regulation of $N$-methylD-aspartate (NMDA) and $\alpha$-amino-3-hydroxy-5-methyl-4-isoxazolepropionic acid (AMPA) receptor complements across the cortex, and to investigate NMDA receptor (NMDAR)-based models of persistent activity, we compared NMDA/AMPA ratios in prefrontal (PFC) and visual cortex (VC) in rat. Whole cell voltage-clamp responses were recorded in brain slices from layer $2 / 3$ pyramidal cells of the medial PFC and VC of rats aged $\mathrm{p} 16-\mathrm{p} 21$. Mixed miniature excitatory postsynaptic currents (mEPSCs) having AMPA receptor (AMPAR)- and NMDAR-mediated components were isolated in nominally $0 \mathrm{Mg}^{2+}$ ACSF. Averaged mEPSCs were well-fit by double exponentials. No significant differences in the NMDA/AMPA ratio (PFC: $27 \pm 1 \%$; VC: $28 \pm 3 \%$ ), peak mEPSC amplitude (PFC: $19.1 \pm 1 \mathrm{pA}$; VC: $17.5 \pm 0.7 \mathrm{pA})$, NMDAR decay kinetics (PFC: $69 \pm 8 \mathrm{~ms}$; VC: $67 \pm 6 \mathrm{~ms}$ ), or degree of correlation between NMDAR- and AMPAR-mediated mEPSC components were found between the areas (PFC: $n=27$; VC: $n=28$ ). Recordings from older rats (p26-29) also showed no differences. EPSCs were evoked extracellularly in $2 \mathrm{mM} \mathrm{Mg}^{2+}$ at depolarized potentials; although the average NMDA/AMPA ratio was larger than that observed for mEPSCs, the ratio was similar in the two regions. In nominally 0 $\mathrm{Mg}^{2+}$ and in the presence of CNQX, spontaneous activation of NMDAR increased recording noise and produced a small tonic depolarization which was similar in both areas. We conclude that this basic property of excitatory transmission is conserved across PFC and VC synapses and is therefore unlikely to contribute to differences in firing patterns observed in vivo in the two regions.
\end{abstract}

\section{IN T R O D U C T I O N}

An important issue in understanding cortical circuits is the regulation of the function and relative number of $N$-methyl-Daspartate (NMDA) and $\alpha$-amino-3-hydroxy-5-methyl-4-isoxazolepropionic acid (AMPA) receptors at excitatory synapses. Because these receptors have distinct kinetics (AMPA, $\tau=$ 2-7 ms; NMDA, $\tau=50-100 \mathrm{~ms}$ ) and because the NMDA receptor is important for $\mathrm{Ca}^{2+}$-dependent plasticity and learning (Bliss and Collingridge 1993; Malenka and Nicoll 1999), the ratio of NMDA receptor (NMDAR) mediated to AMPA receptor (AMPAR) mediated currents at excitatory synapses is likely to be a key determinant of both the short-term integration of synaptic inputs by cortical neurons and their long-term modification. It has been shown that in visual cortical synapses

Address for reprint requests: S. Nelson, Brandeis University, MS 008, 415 South St., Waltham, MA 02454-9110 (E-mail: nelson@brandeis.edu). in vitro, neural activity coregulates the AMPA and NMDA components of excitatory postsynaptic currents (EPSCs) to maintain a constant ratio (Watt et al. 2000). However, little is known as to whether this coupling of NMDARs and AMPARs is regulated in the same way across cortical areas. Although cortical areas can differ in terms of architectonics and cell types, it is not known whether there are regional differences in basic excitatory transmission. Synapses in different cortical areas may be subject to different activity regimes, and synaptic transmission could be tailored to accommodate this, providing a substrate for different processing demands of local circuitry.

Regional variations in the NMDA/AMPA ratio have been proposed to contribute (Compte et al. 2000; Lisman et al. 1998; Wang 1999, 2001) to the ability of prefrontal cortical neurons to exhibit persistent activity, a candidate neural correlate of short-term memory in primates (Chafee and Goldman-Rakic 1998; Fuster and Alexander 1971; Miller et al. 1996; Romo et al. 1999) and rodents (Sakurai and Sugimoto 1986; Schoenbaum and Eichenbaum 1995a,b; Jung et al. 1998; Kesner et al. 1996; Ragozzino and Kesner 2001). The NMDAR is a favored candidate mechanism for sustained recurrent excitation because the slow kinetics aid stability of the memory trace, and the dual activation requirements of depolarization and ligand binding could be used to select a reverberating circuit of only those cells which receive an initial stimulus. Support for such models has so far been indirect. Higher levels of mRNA for NMDARs in prefrontal cortex than in other cortices were found in a human postmortem immunohistological study (Scherzer et al. 1998), and in rats, antagonists for NMDARs, given either systemically (Verma and Moghaddam 1996) or through microinjection of the PFC (Aura and Riekkinen 1999), impair performance on delayed alternation tasks requiring working memory.

Here we attempt to assess a possible specialization of excitatory transmission in the PFC by comparing it to transmission in a functionally and anatomically distinct cortical region using precisely the same methods. The primary visual cortex is a well-studied area in rat, not thought to be involved in working memory processes. Prior studies have measured the NMDA/ AMPA ratio in both visual cortical culture and slices (Umemiya et al. 1999; Watt et al. 2000). In comparison, although specializations in synaptic plasticity have been found in the prefrontal cortex (PFC) (Hempel et al. 2000), less is known about the functional NMDA/AMPA ratio. Burgard and

\footnotetext{
The costs of publication of this article were defrayed in part by the payment of page charges. The article must therefore be hereby marked "advertisement" in accordance with 18 U.S.C. Section 1734 solely to indicate this fact.
} 
Hablitz (1993) assessed the contribution from NMDARs to excitatory synaptic transmission in PFC layer $2 / 3$ pyramidal neurons during early postnatal development (p3-p14). Layer $2 / 3$ is a good candidate for a layer in which reverberatory circuits of recurrent excitation may be found, since it possesses strong lateral excitation (Lewis and Gonzalez-Burgos 2000). Surveying layer $2 / 3$ synapses over a longer developmental range is warranted, since the function of NMDAR at cortical synapses is developmentally regulated, in part by a change in receptor subunit expression which occurs around p14 in visual and somatosensory cortex (Flint et al. 1997; Nase et al. 1999; Sheng et al. 1994). By around p28, many other properties of cortical synapses have largely matured (Reyes and Sakmann 1999).

Therefore to examine the issue of NMDAR and AMPAR regulation across cortical areas, and to explore the hypothesis that NMDARs make a greater contribution to transmission in the PFC, we measured the NMDA/AMPA ratio in layer $2 / 3$ pyramidal neurons in slices from 2- to 4-week-old rats using several complementary approaches. We found that the relative contributions of NMDARs and AMPARs were remarkably conserved between the two cortical regions. The similarity across cortical regions was true for mixed NMDAR- and AMPAR-mediated miniature EPSCS (mEPSCs), for evoked EPSCs, and for spontaneous activation of synaptic and extrasynaptic NMDARs in the absence of AMPAR-mediated transmission. These results indicate that the function of AMPARs and NMDARs are regulated similarly in the supragranular layers of two functionally distinct cortical regions, suggesting a homogeneity of regulation of these receptors across the cortex. Therefore specializations of these regions for particular patterns of activity (such as persistent activity) may more likely reflect regional differences in input, modulation, or circuitry.

\section{METHODS}

All experimental procedures conformed to National Institutes of Health guidelines and were carried out with approval of the Brandeis Animal Care and Use Committee. Long Evans rats, p16-21 and p26-29, were anesthetized with inhalation of isoflurane and decapitated. The brain was quickly removed $(<1 \mathrm{~min})$ and placed immediately into cold (approximately $1^{\circ} \mathrm{C}$ ) and preoxygenated artificial cerebrospinal fluid (ACSF) for approximately $1 \mathrm{~min}$. ACSF contained the following (in mM): $126 \mathrm{NaCl}_{2}, 3 \mathrm{KCl}, 2 \mathrm{MgSO}_{4}, 1 \mathrm{NaH}_{2} \mathrm{PO}_{4}, 25$ $\mathrm{NaHCO}_{3}, 2 \mathrm{CaCl}_{2}, 10$ dextrose. The brain was blocked and submerged in cold ACSF, and $400 \mu \mathrm{M}$ slices were sectioned coronally from either PFC or VC using a Vibratome slicer. Slices contained medial PFC (alternately referred to as anterior cingulate or prelimbic cortex) and primary visual cortex. The anatomic locations of these recordings were assessed with reference to Paxinos and Watson (1986), using the shape of the subcortical white matter as the primary landmark. The anterior/posterior position of PFC slices corresponded to plates 9-11 of the atlas; VC slices corresponded to plates 41-48. About six slices from each hemisphere equilibrated undisturbed at room temperature for at least $1 \mathrm{~h}$, bubbled with $95 \% \mathrm{O}_{2}-5 \% \mathrm{CO}_{2}$, before recording.

\section{Voltage-clamp recording}

Glass micropipettes were pulled from $1.0 \mathrm{~mm}$ outer diameter thinwalled capillary tubing (Warner Instruments) on a Flaming-Brown horizontal puller (Sutter). For mEPSC experiments, internal solution contained the following (in $\mathrm{mM}$ ): $130 \mathrm{~K}$-methylsulfonate or K-gluconate, $10 \mathrm{KCl}, 10 \mathrm{HEPES}, 0.5$ EGTA, 2-3 $\mathrm{Na}_{2} \mathrm{ATP}, 0-1$ guanosine 5 '-triphosphate (GTP), and $2 \mathrm{MgSO}_{4}$. Osmolarity was $280-290 \mathrm{~m} \Omega$ and $\mathrm{pH}$ 7.2-7.4. For evoked EPSC experiments, internal solution contained the following (in $\mathrm{mM}$ ): 130 cesium methanesulfonate, 10 $\mathrm{KCl}, 10$ HEPES, 1 bis-(o-aminophenoxy)- $N, N, N^{\prime}, N^{\prime}$-tetraacetic acid (BAPTA), 2-3 $\mathrm{Na}_{2} \mathrm{ATP}, 0-1 \mathrm{GTP}, 2 \mathrm{MgSO}_{4}, 10$ tetraethylammonium (TEA)-Cl. Whole cell recording pipette resistances were 2.5-5 $\mathrm{M} \Omega$. Pyramidal cells of layers $2 / 3$ were illuminated and visualized using a $\times 40$ water-immersion objective mounted on a fixed-stage phase contrast microscope (Nikon Optiphot UD), and the image was displayed on a video monitor using a charge-coupled device camera. Voltage-clamp recordings were performed using an Axopatch 1D amplifier (Axon Instruments). Signals were filtered at $2 \mathrm{kHz}$. Recordings were made $\leq 8 \mathrm{~h}$ following slicing.

\section{Acquisition}

Signals were sampled at $5-10 \mathrm{kHz}$ and analyzed using the IgorPro (Wavemetrics, Oswego, OR) data acquisition software and customized analysis procedures running on a Macintosh G3 or G4 computer. mEPSCs. Recordings were made in ACSF containing no added $\mathrm{Mg}^{2+}$ to reduce $\mathrm{Mg}^{2+}$ block of NMDARs. Tetrodotoxin $(1 \mathrm{mM})$ and bicuculline $(50 \mu \mathrm{M})$ were added to isolate excitatory mEPSCs. Glycine $(50 \mu \mathrm{M})$, a necessary cofactor for NMDAR activation, was also added. Slices were transferred to the recording chamber from $2 \mathrm{mM}$ $\mathrm{Mg}^{2+} \mathrm{ACSF}$ and equilibrated for $\geq 25 \mathrm{~min}$ before recordings were attempted, to allow the $\mathrm{Mg}^{2+}$ to wash from the slice. Under these conditions, mEPSCs having both an AMPAR- and an NMDARmediated component were readily observed. Ten to twenty 30 -s traces were acquired for each neuron. All recordings were done at room temperature to lower the frequency of events and therefore decrease the likelihood that successive events would occur within the long NMDAR-mediated tails of the mEPSCs. In some cases a second round of acquisition proceeded after bath perfusion of $100 \mu \mathrm{M}$ APV, to isolate AMPA currents.

EVOKED EPSCS. Mixed NMDA/AMPA EPSCs were evoked in 2 $\mathrm{mM} \mathrm{Mg}^{2+}$ ACSF with bicuculline $(50 \mu \mathrm{M})$ and glycine $(50 \mu \mathrm{M})$. The slice was stimulated with a $100-\mu$ s shock delivered through a glass micropipette (tip diameter 10-50 $\mu \mathrm{m}$ ) and placed 50-100 $\mu \mathrm{m}$ lateral to the recording pipette in layer $2 / 3$. Stimulus amplitude was adjusted to produce a single-peaked response with a short constant latency (2-3 $\mathrm{ms}$ ) and an average amplitude of approximately $60 \mathrm{pA}$. Visually identified pyramidal cells were held at $-70 \mathrm{mV}$. Recordings began $\geq 10 \mathrm{~min}$ after initiating whole cell recording, to allow dialysis of the $\mathrm{Cs}^{+} /$TEA internal solution, so that large depolarizing voltage steps could be given while minimizing activation of voltage-dependent $\mathrm{K}^{+}$ currents. Equilibrium of dialysis was seen on average as a stable approximately twofold increase in resting input resistance. Evoked EPSCs were recorded at $-90,0$, and $+50 \mathrm{mV}$, and in some cells intervening voltage steps. EPSCs were evoked at low frequency (once every $10-60 \mathrm{~s}$ ) to minimize accumulation of slowly decaying currents activated during steps to $+50 \mathrm{mV}$. We confirmed that this procedure did not result in long-term potentiation (LTP) of the evoked EPSCs (perhaps because of the initial waiting period of 10-12 min and the inclusion of $1 \mathrm{mM}$ BAPTA in the recording pipette) by comparing the EPSC amplitudes from the beginning and end of the recording period.

NMDA "NOISE." In general, the slow time course and small amplitude of NMDAR-mediated mEPSCs makes it difficult to resolve individual events without the sharp rise time of an accompanying AMPA mEPSC as a "tag." Yet detection using only mEPSCs which also have an AMPA component excludes any all-NMDAR-mediated events ("silent synapses"), as well as contributions from extrasynaptic NMDARs which could also differ in the two regions. To monitor the contribution of NMDARs not accompanied by AMPAR-mediated transmission, we compared epochs of data recorded in no added $\mathrm{Mg}^{2+}$ ACSF containing 6-cyano-7-nitroquinoxaline-2,3-dione (CNQX) (20 $\mu \mathrm{M})$, bicuculline $(50 \mu \mathrm{M})$, glycine $(50 \mu \mathrm{M})$, and TTX $(1 \mathrm{mM})$, before and after blocking NMDARs with APV $(100 \mu \mathrm{M})$. 


\section{Analysis}

Cells were included if $V_{\text {rest }}$ was more hyperpolarized than $-60 \mathrm{mV}$, and $R_{\text {series }} \leq 20 \mathrm{M} \Omega$. Resting $(-70 \mathrm{mV}) R_{\text {input }}$ was monitored throughout the recording; traces in which the resistance changed by $>20 \%$ were excluded. For recordings in normal internal solution, $R_{\text {input }}$ ranged from 150 to $400 \mathrm{M} \Omega$; this was larger with $\mathrm{Cs}^{+}$internal, due to a blockade of $\mathrm{K}^{+}$conductance, with a range of 300-800 $\mathrm{M} \Omega$. $\mathrm{Cs}^{+}$recordings also produced a depolarized $V_{\text {rest }}$; in these cases $V_{\text {rest }}$ was not used as an inclusion criterion.

mEPSCs. Spontaneous events were measured using an in-house mEPSC analysis package which allows for consistent and automated analysis based on preset detection/selection parameters. The decays of average mEPSCs were fit with double-exponential functions. The peak of an mEPSC (taken as the AMPA peak) was measured as the mean current over a 1-ms window. NMDA current amplitude was measured over a 5-ms window from 18 to $23 \mathrm{~ms}$ after the AMPA peak, a time at which AMPA-only mEPSCs $(n=5)$ had declined to $0.26 \pm 0.16 \mathrm{pA}$. The NMDA/AMPA ratio for mEPSCs was given as the ratio of these two measurements.

EVOKED EPSCS. Ten to twenty traces were averaged at each holding potential. Only single peaked, fast latency $(2-3 \mathrm{~ms})$ events were included to ensure monosynaptic responses. Occasional traces containing stimulus-evoked epileptiform events (reflecting inhibitory blockade with $20 \mu \mathrm{M}$ bicuculline) were excluded from the average waveform. Average waveforms from the -90 and +50 $\mathrm{mV}$ holding steps were baseline subtracted and measured using in-house procedures written for IgorPro. The time of the peak current at $-90 \mathrm{mV}$, considered to be fully mediated by AMPAR, was used to establish the time window for measuring the AMPA peak at $+50 \mathrm{mV}$. The decay to baseline of the AMPA current at -90 was used to select a time window for measurement of the NMDA current; a 10-ms measurement window beginning $40 \mathrm{~ms}$ after the stimulus artifact was used. This current was designated as the NMDA measurement. $\left(I_{\mathrm{NMDA}}\right.$ at $+50 \mathrm{mV} / I_{\mathrm{AMPA}}$ at $\left.+50 \mathrm{mV}\right)$ was taken as the NMDA/AMPA ratio.

\section{RES U L T S}

\section{$m E P S C s$}

For rats ages p16-p21, mixed NMDAR- and AMPARmediated mEPSCs were well resolved in nominally $\mathrm{Mg}^{2+}$-free ACSF (Fig. 1A). mEPSC amplitudes varied from our threshold of $7 \mathrm{pA}$ up to $100 \mathrm{pA}$. The average number of mEPSCs collected per cell was $190 \pm 16$. Averaging events produced mEPSC current traces well fit with double exponential functions. The slow component of the mEPSCs was abolished with bath application of $200 \mu \mathrm{M}$ APV (Fig. 1B).

The average mEPSC amplitude did not differ significantly between brain areas (PFC: $19.1 \mathrm{pA} \pm 1$; VC: $17.5 \pm 0.7)$. No statistically significant difference was found in the average NMDA/AMPA ratio between the PFC and VC. Indeed, the grand average mEPSC waveforms from each area (an average of averages from each cell) were quite similar (Fig. 2A). The NMDA/AMPA ratio for the PFC was $27 \%( \pm 1 \%, n=27)$; for the $\mathrm{VC}, 28 \%( \pm 3 \%, n=28)$.

Although the proportion of peak current from NMDARs was indistinguishable in both brain areas, it is possible that a regional difference in NMDAR kinetics-similar to that caused by the developmental change in the relative abundance of NR2B and NR2A (Flint et al. 1997; Nase et al. 1999; Sheng et al. 1994)_could give rise to different functionality in the two areas. However, double exponentials fit to the average

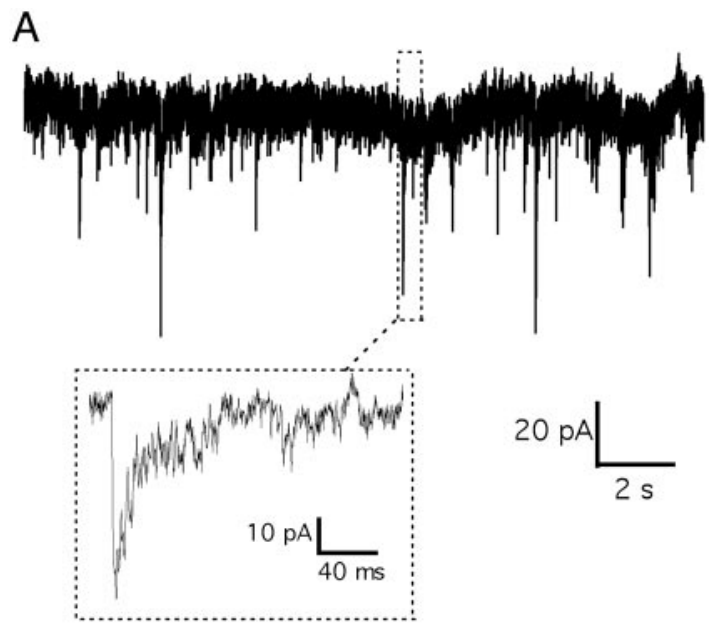

B



FIG. 1. Nominally $\mathrm{Mg}^{2+}$-free bathing medium allows resolution of individual mixed $N$-methyl-D-aspartate receptor (NMDAR) mediated and $\alpha$ amino-3-hydroxy-5-methyl-4-isoxazolepropionic acid receptor (AMPAR) mediated miniature excitatory postsynaptic currents (mEPSCs). A: example trace showing individual events recorded at $-70 \mathrm{mV}$. Inset: a single mixed mEPSC. $B$ : average mEPSC from one neuron recorded before (Control), during (APV), and following washout (Wash) of $100 \mu \mathrm{M} \mathrm{APV}$.

mEPSCs from each cell showed no appreciable difference in decay kinetics between the two areas (Fig. 2Ab).

Although the NMDA/AMPA ratios between areas were indistinguishable, it could be the case that each area has a heterogeneous population of excitatory connections, with varying NMDA/AMPA ratios found across synapses. It is possible that the PFC may have a specialized subset of high-NMDAR synapses which subserve persistent activity, but such regional differences in the proportion of high and low NMDAR synapses may be lost in averaging. Therefore 40 mEPSCs were randomly selected from each cell, and the AMPA peak of each event was plotted against its NMDA measurement. A fit to the data showed a similar degree of correlation of AMPA-toNMDA responses in the two areas (PFC, $r=0.48$; VC, $r=$ 0.57 ; Fig. $2 B$ ). As with the average NMDA/AMPA ratios, the distributions of the NMDA/AMPA ratios of individual events did not significantly differ (Kolmogorov-Smirnov test, $P=$ $0.65)$. The degree of correlation observed is qualitatively similar to findings from Watt et al. (2000). The slightly higher mean correlation of 0.65 reported in that study may reflect the lower number of cells pooled $(n=6)$, a difference between cell types (layer IV in the prior study vs. layer $2 / 3$ in the present study), or a greater degree of variation due to the comparatively smaller NMDA signal in our measurements. 

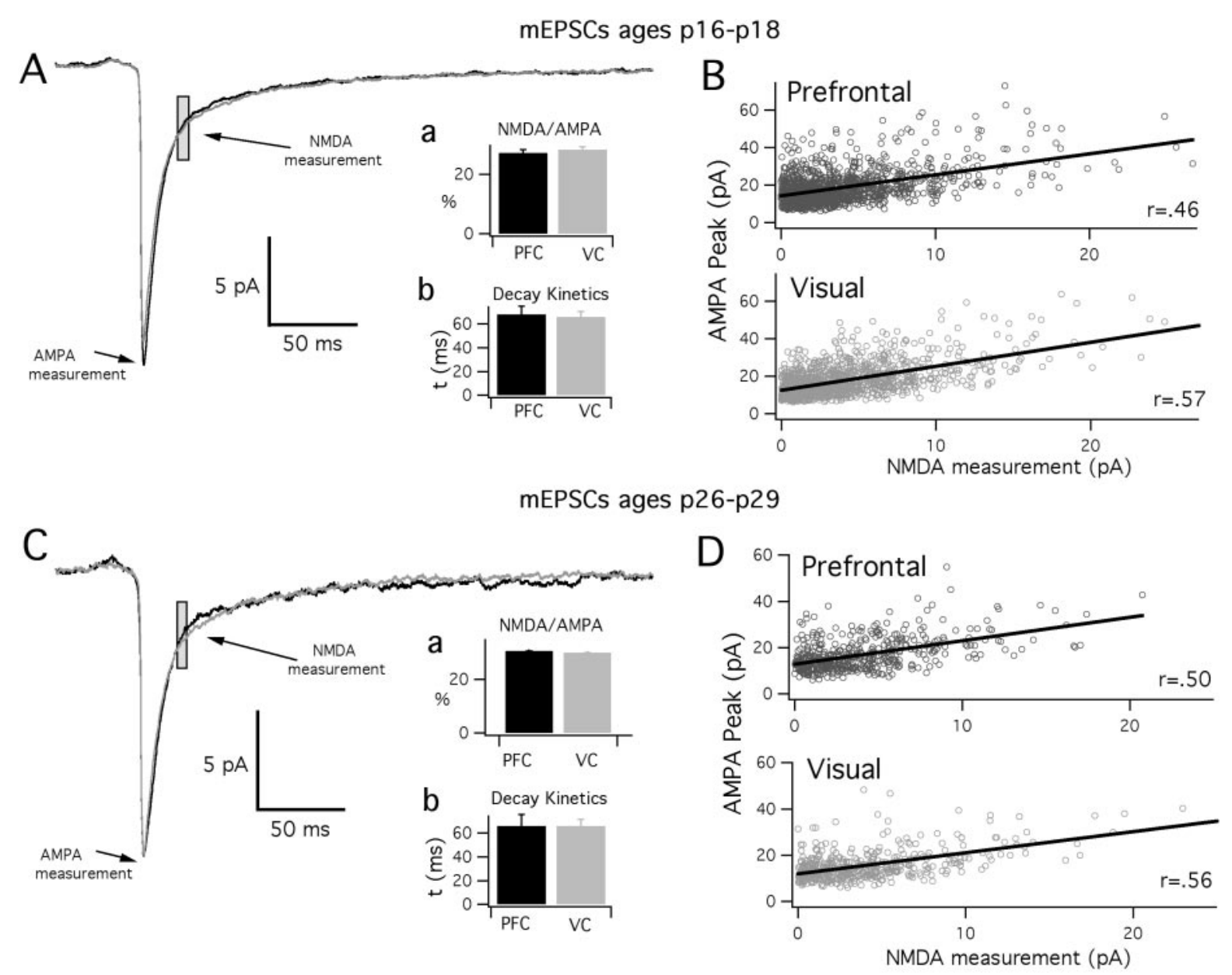

FIG. 2. Mixed mEPSCs from medial prefrontal cortex (PFC) and visual cortex (VC) show similar ratios of NMDAR- to AMPAR-mediated components. $A$ and $B$ : for rats ages p16-p18. $A$ : grand average of $N$-methyl-D-aspartate (NMDA) $/ \alpha$-amino-3hydroxy-5-methyl-4-isoxazolepropionic acid (AMPA) mEPSCs from the mPFC (black trace, $n=28$ cells) and VC (gray trace, $n=$ 27 cells) reveal nearly identical amplitude and kinetics. Insets: $a$ : the NMDA/AMPA ratio and $b$ : average time constants of decay of the NMDA component of the mEPSC for each area. $B$ : correlation between the AMPA and NMDA amplitudes of individual mEPSCs is similar in the two areas. Peak AMPA amplitude vs. NMDA measurement for each individual mEPSC with linear fit. $C$ and $D$ : same as in $A$ and $B$, but for rats ages p26-p29 (PFC, $n=8$; VC, $n=8$ ).

Although the results of the mEPSC analysis showed no difference for ages p16-20, we duplicated the mEPSC experiments using rats from p26-29. Very similar results as those from Fig. 2, $A$ and $B$, are shown in Fig. 2, $C$ and $D$, for the older animals. Again, there were no statistical differences between the two areas, with both having an NMDA/AMPA ratio of approximately $30 \%$ and decay kinetics of approximately 65 $\mathrm{ms}$, essentially the same as with the younger group. Also, the correlations of NMDA versus AMPA measurements were similar to those observed in the p16-20 rats.

\section{Evoked EPSCs}

Analysis of mEPSCs provides the advantage of relatively unbiased sampling from a large number of synapses onto a single cell. However, due to the noisiness of the baseline in 0 $\mathrm{Mg}^{2+}$ recordings, the signal-to-noise ratio is low in comparison with AMPA-only mEPSCs, raising the possibility that small but real differences could be missed. Therefore we corroborated our results by using extracellular stimulation to evoke larger and stimulus-locked EPSCs. Another important advantage is that evoked EPSCs may include currents mediated by NMDA-only synapses.

In $\mathrm{Mg}^{2+}$-free ACSF with bicuculline but no TTX, slices tended to become active and fire frequent epileptic discharges which interfered with clean recordings. Therefore we adopted a different strategy for the evoked EPSCs. Slices were perfused in normal $(2 \mathrm{mM}) \mathrm{Mg}^{2+}$ so that at hyperpolarized potentials most NMDA current was blocked. We assessed the time course of the AMPA component by stimulating while the cell was held at $-90 \mathrm{mV}$. The slice was then stimulated while the cell was held to $+50 \mathrm{mV}$, which provides sufficient depolarization and driving force to reveal the NMDAR component of the EPSC (Fig. 3A). EPSCs reversed at or slightly above $0 \mathrm{mV}$ and were completely blocked by the addition of CNQX and APV (not shown). Averages of 10-20 responses at both -90 and $+50 \mathrm{mV}$ were used to measure the AMPA and NMDA components. As with the mEPSCs, there was no difference in the NMDA/AMPA ratio between the two areas (PFC: $125 \% \pm 6$, $n=9$; VC: $125 \% \pm 7, n=10$ ). This method somewhat overestimates the AMPA component, since there was also a small degree of activation of NMDA at the time of the AMPA 


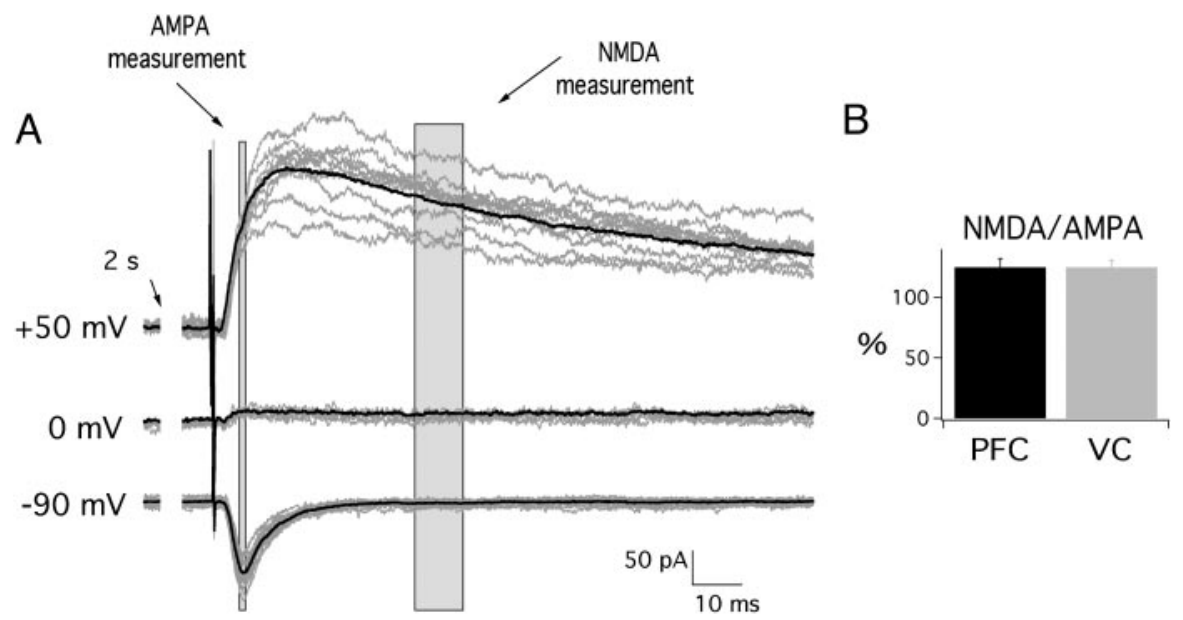

FIG. 3. Average NMDA/AMPA ratios of EPSCs evoked extracellularly in $2 \mathrm{mM} \mathrm{Mg}^{2+}$ do not differ in the 2 areas. $A$ : example traces: extracellular stimulation 50-100 $\mu \mathrm{m}$ lateral to recorded cell evokes EPSCs while cell held to $-90,+10$, and $+50 \mathrm{mV}$. Gray traces indicate repeated trials; black traces show average waveforms. Gray bars indicate windows used to measure peak of AMPAR $(1 \mathrm{~ms})$ and peak of NMDAR $(10 \mathrm{~ms})$-mediated components, both at $+50 \mathrm{mV}$. Stimulation given approximately $2 \mathrm{~s}$ following voltage step to allow voltage-activated currents to return to steady state. $B$ : average NMDA/ AMPA ratios for both areas, with no significant difference.

peak. Measurements from isolated NMDA currents indicate that this accounted for on average $22 \%$ of the measured peak $( \pm 8 \%, n=4)$ and therefore we estimate the NMDA/AMPA ratio at +50 in the evoked condition to be approximately $160 \%$.

\section{Factors contributing to discrepant estimates of the NMDA/AMPA ratio}

The NMDA/AMPA ratio measured from evoked EPSCs (160\%) was much larger than that measured from mEPSCs (30\%). Several methodological differences could account for this discrepancy. First, since we are only evaluating mEPSCs which have an AMPA component, we could be neglecting a large population of NMDA-only (silent) synapses. However, silent synapses in visual cortex are reported to be largely absent by the ages studied (Rumpel et al. 1998).

Second, although we used a nominally $\mathrm{Mg}^{2+}$-free ACSF for the mEPSC recordings, endogenous $\mathrm{Mg}^{2+}$ from the slice, or $\mathrm{Mg}^{2+}$ from the internal solution (from either inside the cell or blown onto the cell during patching), could provide a partial block of NMDARs (Hestrin et al. 1990) sufficient to greatly decrease the NMDA/AMPA ratio. To test this, we recorded evoked NMDA-only EPSCs in a range of $\mathrm{Mg}^{2+}$ concentrations. Although disinhibited by bicuculline, the inclusion of CNQX decreased the likelihood of epileptiform activity (although some epileptiform discharges, presumably mediated solely through NMDARs, did occasionally occur). NMDAonly events were recorded over a range of voltages $(-90,-70$, $-50,-30,0,+30,+50,+70 \mathrm{mV})$, and $I / V$ curves generated for each of four $\mathrm{Mg}^{2+}$ concentrations: $2,1,0.2 \mathrm{mM}$, and no added $\mathrm{Mg}^{2+}$ (example, Fig. $4 A$ ). In all cases, the $I / V$ curve had the classic " $\mathrm{j}$-shape" characteristic of the $\mathrm{Mg}^{2+}$-based voltage dependence (Fig. $4 B$ ). To quantify the degree of $\mathrm{Mg}^{2+}$ block in the no added $\mathrm{Mg}^{2+}$ condition, $I / V$ relations for the known values of $\left[\mathrm{Mg}^{2+}\right]$ were then fit with the equation

$$
I(V)=\frac{g_{\max }\left(V-V_{\text {rev }}\right)}{\left\{1+\left(\left[\mathrm{Mg}^{2+}\right] / K\right) \exp \left(-V / V_{0}\right)\right\}}
$$

where $g_{\max }$ is maximum conductance, $K$ is a $\mathrm{Mg}^{2+}$ binding constant, and $V_{0}$ is the potential at which the linear portion of the curve becomes nonlinear; from Jahr and Stevens (1990). The best fit of this equation to the " 0 " $\mathrm{Mg}^{2+}$ curve gave an estimate of the actual residual $\mathrm{Mg}^{2+}, 0.03( \pm 0.01) \mathrm{mM}$. To test

the validity of this estimate, we recorded mEPSCs in $0.03 \mathrm{mM}$ $\mathrm{Mg}^{2+}$. The average waveforms of the two $\mathrm{Mg}^{2+}$ conditions (no added $\mathrm{Mg}^{2+}$ and $0.03 \mathrm{mM} \mathrm{Mg}^{2+}$ ) were indistinguishable (Fig. 4C).

To estimate the reduction of the NMDA component in mEPSCs due to residual $\mathrm{Mg}^{2+}$, we fitted a line to all data points for all voltage steps more positive than $0 \mathrm{mV}$ (dashed line, Fig. $4 B$ ) and measured the difference in current at $-70 \mathrm{mV}$ on this line and at the corresponding point on the " 0 " $\mathrm{Mg}^{2+}$ curve. This comparison showed that the $0 \mathrm{Mg}^{2+}$ curve evaluated at $-70 \mathrm{mV}$ was reduced to $62 \%$ of what it would have been had the NMDA $I / V$ relation been linear (i.e., no $\mathrm{Mg}^{2+}$ block). Taking this into account, the corrected estimate should put the NMDA/AMPA ratio for the mEPSCs at $45 \%$ for both the PFC and VC.

Just as the NMDA/AMPA ratio may have been underestimated in the mEPSCs due to residual $\mathrm{Mg}^{2+}$, it is possible we may have overestimated the NMDA/AMPA ratio of the evoked EPSCs. One way this could happen is if there was greater dendritic filtering in the evoked EPSCs, which would tend to diminish the faster AMPA events more than the slow NMDA events. We do not think this is likely, since the average EPSC rise times at $-90 \mathrm{mV}$ and the NMDA/AMPA ratios were poorly correlated $(r=-0.31)$, and the NMDA/AMPA ratios from cells with the fastest average AMPA rises $(<1.5 \mathrm{~ms}, n=$ 9 ; average ratio $=127 \pm 7 \%$ ) were not on average different from the ratios of the remaining cells $(>1.5 \mathrm{~ms}, n=10$; average ratio $=123 \pm 7 \% ; P=0.3$ ), suggesting that filtering does not account for the large NMDA/AMPA ratio in the evoked EPSCs. Another possible cause for overestimating the ratio is if the AMPA component were inwardly rectifying at depolarized potentials (Kumar and Huguenard 2001). To test this, we produced an AMPA-only $I / V$ curve (in APV and bicuculline) over the same range of voltages given above. Beginning around $+30 \mathrm{mV}$ there was on average some inward rectification (example, Fig. 4D). We measured the inward rectification at $+50 \mathrm{mV}$ (the voltage at which the NMDA/ AMPA ratio had been assessed in the EPSCs) and found the response diminished to $68 \%$, based on a linear fit of the AMPA conductance at hyperpolarized potentials (dashed line, Fig. $4 D$ ). Since the AMPA component was diminished to $68 \%$ of maximum, this corrects the NMDA/AMPA ratio for the evoked EPSCs from 160 to $109 \%$.

These two methodological corrections-residual $\mathrm{Mg}^{2+}$ and 


\section{A NMDA voltage dependence in no added $\mathrm{Mg}++$}

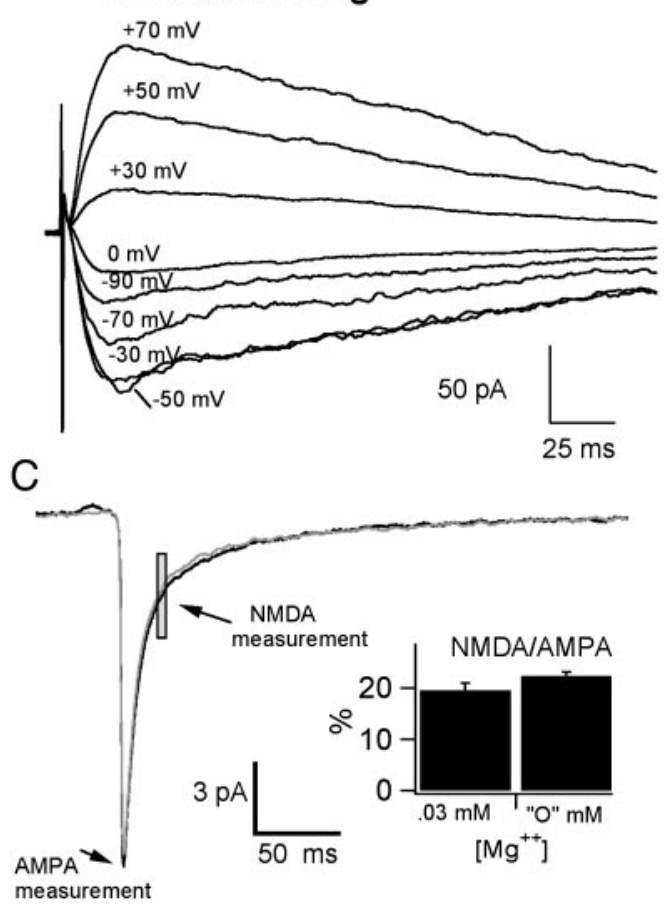

B NMDA I/Vs in Varying Mg++
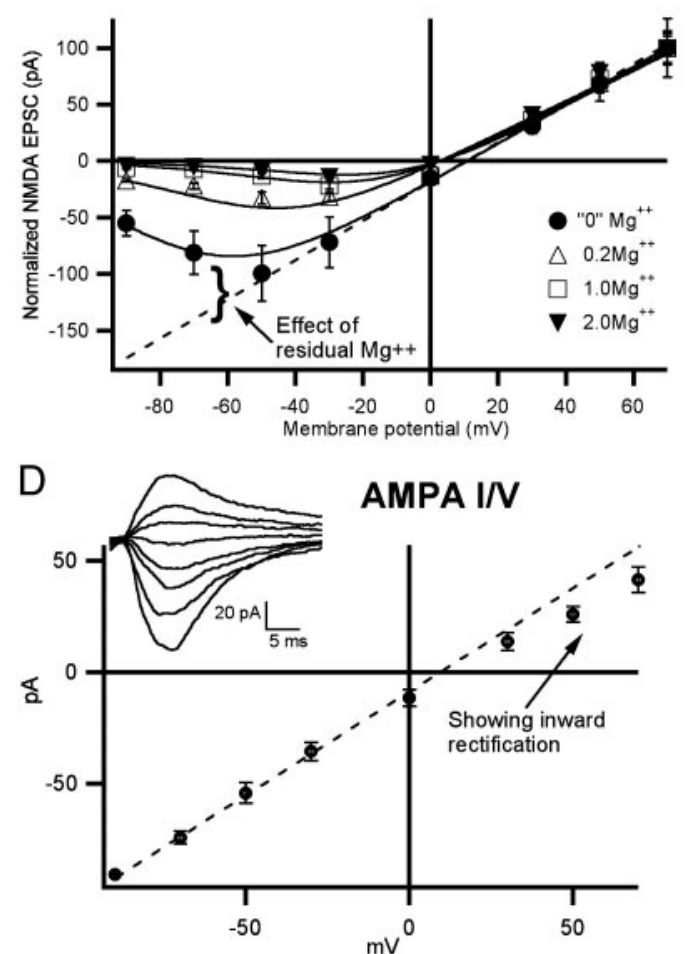

FIG. 4. Voltage dependence of NMDARs in nominally $0 \mathrm{Mg}^{2+}$ and inward rectification of AMPARs contribute to overestimation of the NMDA/AMPA ratio in evoked EPSCs at $+50 \mathrm{mV}$. A: example traces: NMDA-only EPSCs evoked by extracellular stimulation 50-100 $\mu \mathrm{M}$ lateral, at various holding potentials. $B: I / V$ relations for NMDA-only responses (p16-18) over a range of $\mathrm{Mg}^{2+}$ concentrations show a voltage dependence (characteristic "j-shaped" curve) even in no added $\mathrm{Mg}^{2+}$, suggesting a partial block of NMDARs by residual or endogenous $\mathrm{Mg}^{2+}$. From a global fit of $E q .1$ to the known concentrations of $\mathrm{Mg}^{2+}$ we estimated the no added $\mathrm{Mg}^{2+}$ to be $0.03 \mathrm{mM}$. C: average mEPSCs from visual cortex using $0.03 \mathrm{mM}$ and no added $\mathrm{Mg}^{2+}$ show identical waveforms; inset: bar graph with NMDA/AMPA ratio for both concentrations $(0.03 \mathrm{mM}, n=6 ; 0 \mathrm{mM}, n=27)$. $D: I / V$ relation for AMPA-only responses $(n=5)$ showed an inward rectification of responses at depolarized potentials. Inset: example traces of AMPA responses over the range of holding potentials.

inward rectification of AMPA at depolarized potentials-lessens the discrepancy between the NMDA/AMPA ratio as measured with mEPSCs versus evoked EPSCs, but still leaves a difference of approximately $45 \%$ versus approximately $109 \%$. A number of other factors may account for the remaining discrepancy and are explored in DISCUSSION.

\section{NMDA “noise”}

Although the mEPSCs and EPSCs suggest an equal NMDA/ AMPA ratio in PFC and VC, these techniques do not assess possible activation of extrasynaptic NMDA receptors by ambient glutamate or glutamate spillover (Clark et al. 1997; Rusakov and Kullmann 1998). Previous studies have shown that in $0 \mathrm{Mg}^{2+}$ NMDARs contribute a tonic, inward, noisy current in cortical pyramidal neurons (LoTurco et al. 1990). We compared this small "NMDA noise" in the PFC and VC as a means to capture all NMDA conductances: at mixed NMDA and AMPA synapses, silent synapses, and extrasynaptic NMDARs. If there is increased extrasynaptic NMDAR activation in the PFC, this assessment should capture it. We found that bath application of the highly specific NMDAR blocker APV $(100 \mu \mathrm{M})$ reduced noise and abolished a tonic inward current (Fig. 5A). However, the change in the average holding current after APV was similar in both areas (PFC: $-5.9 \pm 1.0$
pA, $n=7$; VC: $-4.8 \pm 1.5 \mathrm{pA}, n=5)$ and not statistically different (Fig. 5B). The noise, measured as the SD of the fluctuating inward current at $-70 \mathrm{mV}$, was also decreased to a similar degree in both brain areas. This suggests that extrasynaptic NMDARs and silent synapses are not differentially distributed between PFC and VC layer 2/3 at this age.

\section{I S C U S S I O N}

For rat layer $2 / 3$ pyramidal cells, we find no statistical difference in NMDA/AMPA ratios between the PFC and VC. This is true whether measured in mEPSCs recorded in nominally $\mathrm{Mg}^{2+}$-free ACSF or in evoked EPSCs at strongly depolarized membrane potentials. We also do not find any difference in the tonic, inward, noisy current attributable to NMDARs. In addition, the degree of correlation between the amplitude of AMPA peaks and NMDA measurements is similar in the two areas. Together these results suggest that these functionally disparate regions are homogeneous with respect to the relative contribution of NMDAR and AMPAR to excitatory transmission in layer $2 / 3$.

The NMDA/AMPA ratio was markedly different depending on whether mEPSCs or evoked EPSCs were assessed. This discrepancy might be accounted for in several ways. First, due to our method of mEPSC detection, only those mEPSCs which 
A

\section{$100 \mu \mathrm{M}$ APV}

Prefrontal Cortex
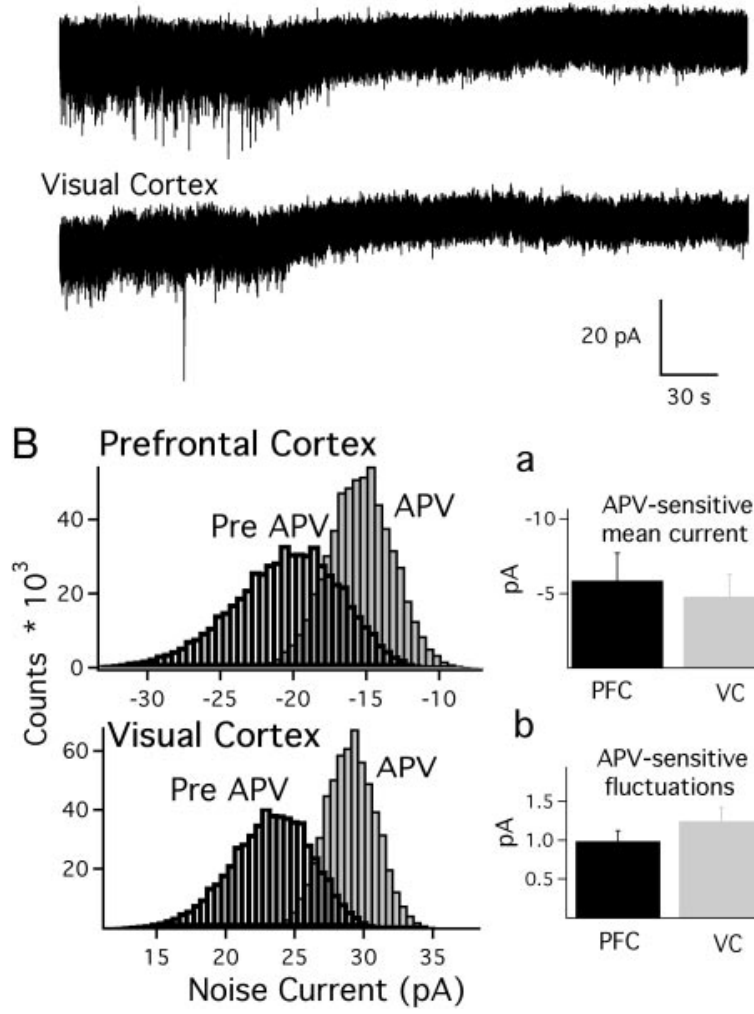

a

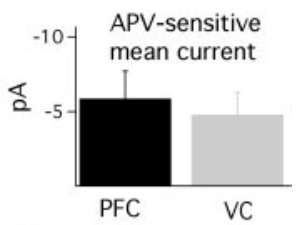

b

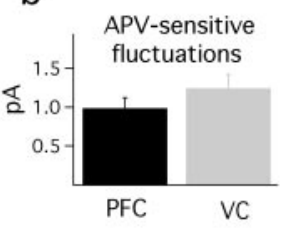

FIG. 5. NMDAR-dependent fluctuating background currents present at $-70 \mathrm{mV}$ in nominally $\mathrm{Mg}^{2+}$-free ACSF are similar in both areas. A: examples from both areas of NMDA-only mEPSCs/"noise" recorded in no added $\mathrm{Mg}^{2+}$ ACSF, which is abolished by perfusion of APV. $B$ : all points histograms from the examples in $A$ show a similar reduction of both the mean and the fluctuations of the inward current for both brain areas. Insets: average reductions in mean and SD of holding current for PFC $(n=5)$ and VC $(n=5)$ following APV.

have an AMPA component ("AMPA tagged") are selected, because slow rise times make NMDA-only events difficult to detect. Therefore we are excluding any silent synapses, which could account for more NMDA current measured with evoked EPSCs. Silent synapses would work particularly well in NMDA-based models of persistent activity, but we think it unlikely that this accounts for the discrepancy. Studies characterizing the development of silent synapses in the visual cortex (Rumpel et al. 1998) find a general elimination of silent synapses in VC by p14. Nevertheless, small-AMPA/bigNMDA mEPSCs may also be missed and cause an underestimate of the NMDA contribution. Another difference between the mEPSCs and evoked EPSC recordings is that the latter were recorded with internal solutions containing high cesium, which may diminish sensitivity of NMDARs to $\mathrm{Mg}^{2+}$ blockade (Qian et al. 2002). However, this is unlikely to account for the observed difference, since $\mathrm{Mg}^{2+}$ blockade is largely absent at the depolarized voltage $(+50 \mathrm{mV})$ used.

More probable factors giving rise to the discrepancy are those which directly reflect properties of spontaneous vesicle fusion at individual synapses versus release evoked (nearly) synchronously at many synapses. Recruitment of a large subset of synapses onto a postsynaptic cell during extracellular stim- ulation may result in glutamate spillover which can contribute to the NMDA component in the EPSCs (Clark et al. 1997; Rusakov and Kullmann 1998). Also, variations in onset time of component EPSCs (due to variations in synaptic delays, axonal path lengths, and fiber recruitment thresholds) can smear out the time course of compound EPSCs relative to that of single release site events. Such smearing out of the response may favor summation of the far slower NMDA component, thereby artificially inflating the NMDA/AMPA ratio. Consistent with this possibility, evoked EPSCs had much longer durations and slower rise times than mEPSCs.

For these two reasons we suspect that the NMDA/AMPA ratios measured with mEPSCs or EPSCs are likely to be different in the way we have observed. Indeed, a review of the related literature seems to indicate this. For the purposes of close comparison with other studies, we have generated a partial survey of reported NMDA/AMPA ratios and our ratios in Table 1, along with other relevant parameters. In the final column of the table we have attempted to normalize the various findings by using the reported parameters and Eq. $l$ to estimate the ratio of maximum conductance of NMDA to AMPA (given as $g_{\max }$ ratio). Interestingly, as in our study, ratios assessed with mEPSCs tend to be smaller than those assessed in pairs or evoked EPSCs, with two qualifications. First, in evoked current-clamp recordings (first 2 rows) $g_{\max }$ ratio values are notably higher. This may reflect the fact that the somatic and subsynaptic membrane may be further from isopotential during current-clamp recordings. Second, in the mEPSC data, there may be an age dependence to the $g_{\max }$ ratio, in that the first two rows show a larger $g_{\max }$ ratio but are sampled in younger animals (p1-p15). Generally, the range of NMDA/AMPA ratios reported is consistent with our findings.

A number of factors are relevant to the applicability of these results to NMDA-based models of persistent activity. First, it is not clear that the same mechanisms or structures are implemented in primate and rodent PFC; for example, layer 4 is found in the PFC of primates but not in rodents. Yet there is evidence for the prefrontal cortex as important for working memory in rat as with monkey (e.g., that PFC lesions in rat impair performance on delayed alternation tasks requiring working memory) (Sakurai and Sugimoto 1985). Although persistent activity and delay-tuned cells have best been explored in the PFC of primates, growing evidence in rat PFC links delay period firing and items held on-line in memory (Jung et al. 1998; Kesner et al. 1996; Ragozzino and Kesner 2001; Sakurai and Sugimoto 1986; Schoenbaum and Eichenbaum 1995a,b).

We confined our recordings to layer $2 / 3$ pyramidal cells. Superficial layers of the cortex have rich local recurrent excitation (Lewis and Gonzalez-Burgos 2000) and as such may be good candidates for local reverberatory working memory circuits. However, it is possible that excitatory synapses of layer $2 / 3$ are not those involved in recurrent excitation leading to persistent activity. Also, NMDAR- and AMPAR-mediated currents have been shown to be independently regulated by a variety of neuromodulatory agents (Aramakis and Metherate 1998; Arvanov et al. 1997; Arvanov and Wang 1998; Castro et al. 1999; Seamans et al. 2001). Future experiments could investigate the effect of such substances on the NMDA/AMPA ratio as measured in mixed NMDA/AMPA mEPSCs or EPSCs in PFC and elsewhere. 
TABLE 1. Review of NMDA/AMPA ratios previously reported

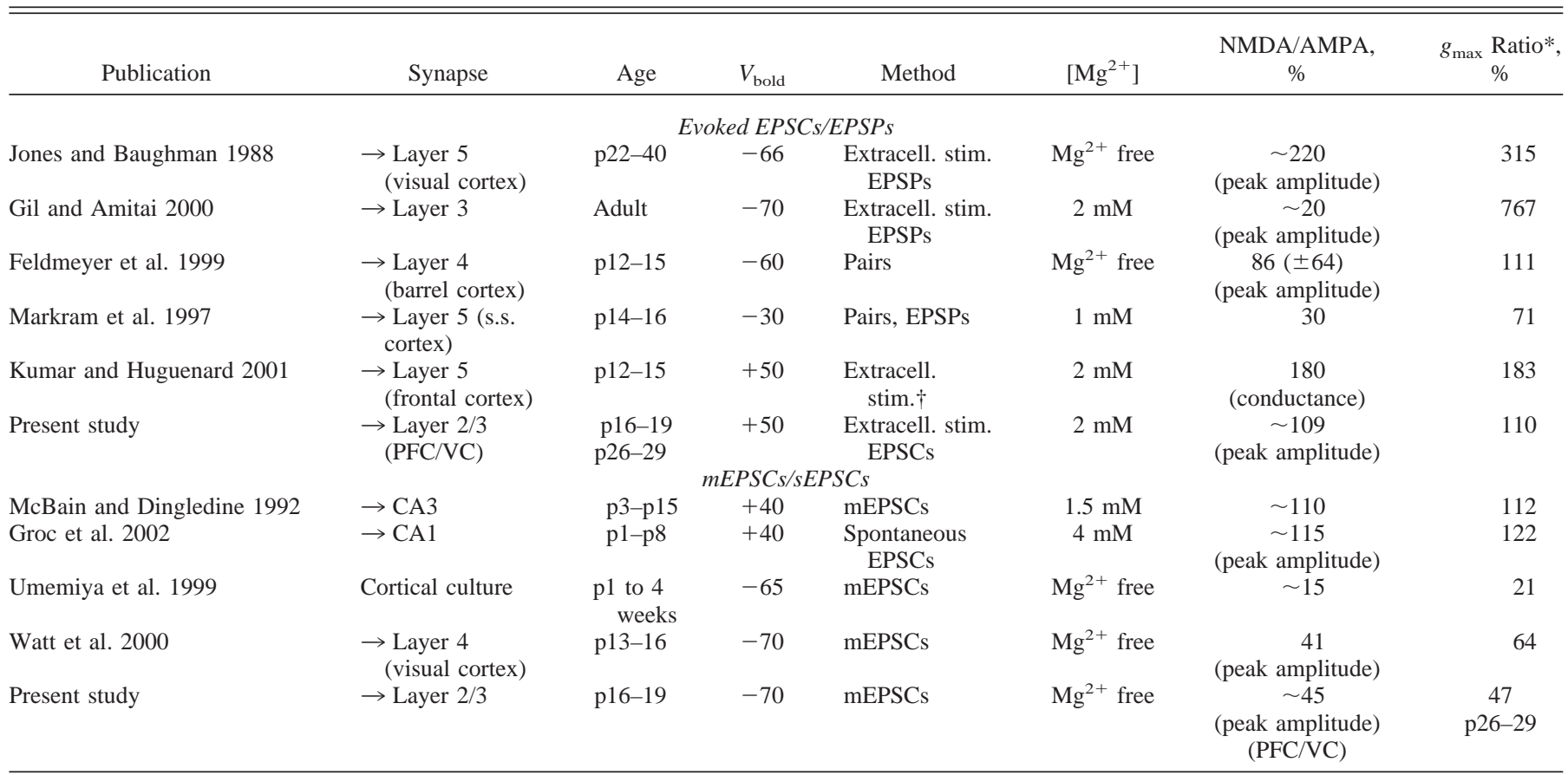

NMDA, $N$-methyl-D-aspartate; AMPA, $\alpha$-amino-3-hydroxy-5-methyl-4-isoxazolepropionic acid; $V_{\text {hold }}$, holding potential; $g_{\text {max }}$, maximum conductance; EPSC, excitatory postsynaptic current; EPSP, excitatory postsynaptic potential; PFC, prefrontal cortex; VC, visual cortex; mEPSCs, miniature EPSCs; sEPSCs, spontaneous EPSCs. $* g_{\max }$ ratio values show the predicted ratio of maximal NMDA and AMPA conductances using Eq. 1 and reported values. ${ }^{\dagger}$ In 0.05 mM NBQX.

The similarity of layer 2/3 NMDA contribution in PFC and VC does not support claims that associative areas specialized for persistent activity should have a greater contribution from NMDARs than primary sensory areas. However, the large NMDA/AMPA ratio (approximately 109\%) in the evoked EPSCs suggests there is ample NMDA conductance in both areas to provide stability of the memory trace in the models. Therefore NMDARs may be abundant throughout the cortex and persistent activity may indeed be maintained by recurrent excitation via NMDAR-mediated current; however, whether such activity is seen may be strongly dependent on the local architecture. Miller and Cohen (2001) have characterized these two accounts of persistent activity as "cellular" versus "circuitbased." Although the PFC has been generally targeted as a key region for working memory, recent results showing delay period activity in primary visual cortex of monkey during a visual task (Super et al. 2001) raise the possibility that comparable and large NMDA contributions in the VC as well as PFC may contribute to persistent activity in both regions.

We thank A. Compte, N. Desai, K. MacLeod, S. Moore, N. Otmakhova, P. J. Sjöström, M. van Rossum, P. Taneja, X.-J. Wang, and A. Watt for help and useful discussions.

\section{I S C L O S URES}

This work was supported by National Institutes of Health Grants RO1 EY-11116 and RO1 NS-36853.

\section{REFERENCES}

Aramakis VB and Metherate R. Nicotine selectively enhances NMDA receptor-mediated synaptic transmission during postnatal development in sensory neocortex. J Neurosci 18: 8485-8495, 1998.
Arvanov VL, Liang X, Schwartz J, Grossman S, and Wang RY. Clozapine and haloperidol modulate $N$-methyl-D-aspartate- and non- $N$-methyl-D-aspartate receptor-mediated neurotransmission in rat prefrontal cortical neurons in vitro. J Pharmacol Exp Ther 283: 226-234, 1997.

Arvanov VL and Wang RY. M100907, a selective 5-HT2A receptor antagonist and a potential antipsychotic drug, facilitates $N$-methyl-D-aspartatereceptor mediated neurotransmission in the rat medial prefrontal cortical neurons in vitro. Neuropsychopharmacology 18: 197-209, 1998.

Aura J and Riekkinen P Jr. Blockade of NMDA receptors located at the dorsomedial prefrontal cortex impairs spatial working memory in rats. Neuroreport 10: 243-248, 1999.

Bliss TV and Collingridge GL. A synaptic model of memory: long-term potentiation in the hippocampus. Nature 361: 31-39, 1993.

Burgard EC and Hablitz JJ. Developmental changes in NMDA and nonNMDA receptor-mediated synaptic potentials in rat neocortex. $J$ Neurophysiol 69: 230-240, 1993.

Castro NG, de Mello MC, de Mello FG, and Aracava Y. Direct inhibition of the $N$-methyl-D-aspartate receptor channel by dopamine and (+)SKF38393. Br J Pharmacol 126: 1847-1855, 1999.

Chafee MV and Goldman-Rakic PS. Matching patterns of activity in primate prefrontal area $8 \mathrm{a}$ and parietal area 7ip neurons during a spatial working memory task. J Neurophysiol 79: 2919-2940, 1998.

Clark BA, Farrant M, and Cull-Candy SG. A direct comparison of the single-channel properties of synaptic and extrasynaptic NMDA receptors. J Neurosci 17: 107-116, 1997.

Compte A, Brunel N, Goldman-Rakic PS, and Wang XJ. Synaptic mechanisms and network dynamics underlying spatial working memory in a cortical network model. Cereb Cortex 10: 910-923, 2000.

Feldmeyer D, Egger V, Lubke J, and Sakmann B. Reliable synaptic connections between pairs of excitatory layer 4 neurones within a single "barrel" of developing rat somatosensory cortex. J Physiol 521(Pt. 1): 169-190, 1999.

Flint AC, Maisch US, Weishaupt JH, Kriegstein AR, and Monyer $\mathrm{H}$. NR2A subunit expression shortens NMDA receptor synaptic currents in developing neocortex. J Neurosci 17: 2469-2476, 1997.

Fuster JM and Alexander GE. Neuron activity related to short-term memory. Science 173: 652-654, 1971.

Gil $\mathbf{Z}$ and Amitai Y. Evidence for proportional synaptic scaling in neocortex of intact animals. Neuroreport 11: 4027-4031, 2000. 
Groc L, Gustafsson B, and Hanse E. Spontaneous unitary synaptic activity in CA1 pyramidal neurons during early postnatal development: constant contribution of AMPA and NMDA receptors. J Neurosci 22: 5552-5562, 2002.

Hempel CM, Hartman KH, Wang XJ, Turrigiano GG, and Nelson SB. Multiple forms of short-term plasticity at excitatory synapses in rat medial prefrontal cortex. J Neurophysiol 83: 3031-3041, 2000.

Hestrin S, Nicoll RA, Perkel DJ, and Sah P. Analysis of excitatory synaptic action in pyramidal cells using whole-cell recording from rat hippocampal slices. J Physiol 422: 203-225, 1990.

Jahr CE and Stevens CF. A quantitative description of NMDA receptorchannel kinetic behavior. J Neurosci 10: 1830-1837, 1990.

Jones KA and Baughman RW. NMDA- and non-NMDA-receptor components of excitatory synaptic potentials recorded from cells in layer $\mathrm{V}$ of rat visual cortex. J Neurosci 8: 3522-3534, 1988.

Jung MW, Qin Y, McNaughton BL, and Barnes CA. Firing characteristics of deep layer neurons in prefrontal cortex in rats performing spatial working memory tasks. Cereb Cortex 8: 437-450, 1998.

Kesner RP, Hunt ME, Williams JM, and Long JM. Prefrontal cortex and working memory for spatial response, spatial location, and visual object information in the rat. Cereb Cortex 6: 311-318, 1996.

Kumar SS and Huguenard JR. Properties of excitatory synaptic connections mediated by the corpus callosum in the developing rat neocortex. J Neurophysiol 86: 2973-2985, 2001.

Lewis DA and Gonzalez-Burgos G. Intrinsic excitatory connections in the prefrontal cortex and the pathophysiology of schizophrenia. Brain Res Bull 52: 309-317, 2000 .

Lisman JE, Fellous JM, and Wang XJ. A role for NMDA-receptor channels in working memory. Nat Neurosci 1: 273-275, 1998.

LoTurco JJ, Mody I, and Kriegstein AR. Differential activation of glutamate receptors by spontaneously released transmitter in slices of neocortex. Neurosci Lett 114: 265-271, 1990.

Malenka RC and Nicoll RA. Long-term potentiation-a decade of progress? Science 285: 1870-1874, 1999.

Markram H, Lubke J, Frotscher M, Roth A, and Sakmann B. Physiology and anatomy of synaptic connections between thick tufted pyramidal neurones in the developing rat neocortex. J Physiol 500: 409-440, 1997.

McBain C and Dingledine R. Dual-component miniature excitatory synaptic currents in rat hippocampal CA3 pyramidal neurons. J Neurophysiol 68 16-27, 1992.

Miller EK and Cohen JD. An integrative theory of prefrontal cortex function. Annu Rev Neurosci 24: 167-202, 2001.

Nase G, Weishaupt J, Stern P, Singer W, and Monyer H. Genetic and epigenetic regulation of NMDA receptor expression in the rat visual cortex. Eur J Neurosci 11: 4320-4326, 1999.

Paxinos G, and Watson C. The Rat Brain in Stereotaxic Coordinates (2nd ed.), San Diego, CA: Academic, 1986.

Qian A, Antonov SM, and Johnson JW. Modulation by permeant ions of $\mathrm{Mg}(2+)$ inhibition of NMDA-activated whole-cell currents in rat cortica neurons. J Physiol 538: 65-77, 2002.
Ragozzino ME and Kesner RP. The role of rat dorsomedial prefrontal cortex in working memory for egocentric responses. Neurosci Lett 308: 145-148, 2001

Reyes A and Sakmann B. Developmental switch in the short-term modification of unitary EPSPs evoked in layer 2/3 and layer 5 pyramidal neurons of rat neocortex. J Neurosci 19: 3827-3835, 1999.

Romo R, Brody CD, Hernandez A, and Lemus L. Neuronal correlates of parametric working memory in the prefrontal cortex. Nature 399: 470-473, 1999.

Rumpel S, Hatt H, and Gottmann K. Silent synapses in the developing rat visual cortex: evidence for postsynaptic expression of synaptic plasticity. J Neurosci 18: 8863-8874, 1998.

Rusakov DA and Kullmann DM. Extrasynaptic glutamate diffusion in the hippocampus: ultrastructural constraints, uptake, and receptor activation. J Neurosci 18: 3158-3170, 1998.

Sakurai Y and Sugimoto S. Effects of lesions of prefrontal cortex and dorsomedial thalamus on delayed go/no-go alternation in rats. Behav Brain Res 17: 213-219, 1985.

Sakurai Y and Sugimoto S. Multiple unit activity of prefrontal cortex and dorsomedial thalamus during delayed go/no-go alternation in the rat. Behav Brain Res 20: 295-301, 1986.

Scherzer CR, Landwehrmeyer GB, Kerner JA, Counihan TJ, Kosinski CM, Standaert DG, Daggett LP, Velicelebi G, Penney JB, and Young AB. Expression of $N$-methyl-D-aspartate receptor subunit mRNAs in the human brain: hippocampus and cortex. J Comp Neurol 390: 75-90, 1998.

Schoenbaum G and Eichenbaum H. Information coding in the rodent prefrontal cortex. II. Ensemble activity in orbitofrontal cortex. J Neurophysiol 74: 751-762, 1995a.

Schoenbaum G and Eichenbaum H. Information coding in the rodent prefrontal cortex. I. Single-neuron activity in orbitofrontal cortex compared with that in pyriform cortex. J Neurophysiol 74: 733-750, 1995 b.

Seamans JK, Durstewitz D, Christie BR, Stevens CF, and Sejnowski TJ. Dopamine D1/D5 receptor modulation of excitatory synaptic inputs to layer V prefrontal cortex neurons. Proc Natl Acad Sci USA 98: 301-306, 2001.

Sheng M, Cummings J, Roldan LA, Jan YN, and Jan LY. Changing subunit composition of heteromeric NMDA receptors during development of rat cortex. Nature 368: 144-147, 1994.

Super H, Spekreijse H, and Lamme VA. A neural correlate of working memory in the monkey primary visual cortex. Science 293: 120-124, 2001.

Umemiya M, Senda M, and Murphy TH. Behaviour of NMDA and AMPA receptor-mediated miniature EPSCs at rat cortical neuron synapses identified by calcium imaging. J Physiol 521(Pt. 1): 113-122, 1999.

Verma A and Moghaddam B. NMDA receptor antagonists impair prefrontal cortex function as assessed via spatial delayed alternation performance in rats: modulation by dopamine. J Neurosci 16: 373-379, 1996.

Wang XJ. Synaptic basis of cortical persistent activity: the importance of NMDA receptors to working memory. J Neurosci 19: 9587-9603, 1999.

Wang XJ. Synaptic reverberation underlying mnemonic persistent activity. Trends Neurosci 24: 455-463, 2001.

Watt AJ, van Rossum MC, MacLeod KM, Nelson SB, and Turrigiano GG. Activity coregulates quantal AMPA and NMDA currents at neocortical synapses. Neuron 26: 659-670, 2000. 